\title{
Estudo de toxicologia clínica de um fitoterápico a base de associações de plantas, mel e própolis
}

\author{
Janaina P. Tavares*, Ismael L. Martins, Ariane S. Vieira, Francisco A.V. Lima, Fernando A.F. \\ Bezerra, Manoel O. Moraes, Maria E.A. Moraes
}

Unidade de Farmacologia Clínica, Departamento de Fisiologia e Farmacologia, Faculdade de Medicina, Universidade Federal do Ceará, Rua Cel. Nunes de Melo 1127, Rodolfo Teófilo, 60431-970, Fortaleza, CE, Brasil

\begin{abstract}
RESUMO: O estudo objetivou avaliar a segurança clínica do uso de uma formulação fitoterápica composta da associação de Mikania glomerata, Mentha piperita, Eucalyptus globulus e Copaifera multijuga, incorporadas à própolis e mel para tratar doenças respiratórias. O ensaio clínico consistiu de um estudo aberto, com 26 voluntários adultos de ambos os sexos, que receberam quatro doses orais de $15 \mathrm{~mL}$ de Saratosse ${ }^{\circledR}$, durante 28 dias ininterruptos. Os voluntários foram incluídos somente se considerados saudáveis, após exames clínico e complementares que antecederam o estudo. A avaliação laboratorial incluía análises hematológicas, bioquímicas e sorológicas. A mesma avaliação foi repetida após cada semana de tratamento e no pós-estudo, sete dias após a última administração. Os resultados foram submetidos à Análise de Variância (teste de Dunnett), obtendo-se, a menor diferença significante $(p<0,05)$. O xarope foi bem tolerado. Alguns eventos adversos foram relatados, sendo classificados como possivelmente ou não atribuídos ao fitoterápico. Os índices de hemoglobina, TGO, TGP, creatinina e leucócitos não apresentaram diferenças significativas em relação ao pré-estudo. Todos os parâmetros laboratoriais estiveram dentro das suas respectivas faixas de normalidade. Os exames clínicos, eletrocardiográficos e laboratoriais não evidenciaram sinais de toxicidade nos órgãos e sistemas avaliados.
\end{abstract}

Unitermos: Farmacologia, toxicidade de drogas, fitoterapia, ensaios clínicos.

ABSTRACT: "Clinical toxicology study of a phytomedicine syrup composed of plants, honey and propolis". The aim of this study is to evaluate the safety of the use of a phytomedicine syrup (Saratosse ${ }^{\circledR}$ ) composed of several medicinal plants: Mikania glomerata, Mentha piperita, Eucalyptus globulus and Copaifera multijuga, along with honey and propolis to treat respiratory diseases. The clinical trial consisted of an open study with 26 adult volunteers of both sexes, who were given an oral dose of $15 \mathrm{~mL}$ of Saratoss $\mathrm{e}^{\circledR}$ for 28 consecutive days, four times a day. Only volunteers who were found healthy after a clinical and physical examination were included. The laboratory tests included: hematological, biochemical and serological analysis. This evaluation was repeated after each week of treatment and seven days after the last administration. ANOVA analysis (Dunnett's test) showed a significant difference, albeit at low statistical level $(\mathrm{p}<0.05)$. Significant differences to the pre-study were not shown by hemoglobin, SGOT, SGPT, creatinine and leukocytes analysis. Laboratorial tests results were within their maximum and minimum reference values. On the whole the medicine was well tolerated. Some side effects were related, which may or not be attributed to the phytomedicine. Clinical, electrocardiographic and laboratory tests did not show any evidence of toxic signs in the organs and systems studied.

Keywords: Pharmacology, toxicity of drugs, phytotherapy, clinical trials.

\section{INTRODUÇÃO}

O xarope Saratosse ${ }^{\circledR}$ é indicado pelo seu fabricante para o tratamento de algumas patologias do trato respiratório. É composto pela associação de várias plantas medicinais, entre as quais a Mikania glomerata, Mentha piperita, Eucalyptus globulus e Copaifera multijuga, incorporadas ao mel e à própolis. Os componentes da formulação são tradicionalmente utilizados pela população devido às propriedades antitussígenas, antisépticas e broncodilatadoras que lhes são atribuídas, entre outras (Reis et al., 2000; Pereira et al., 2004).
Sabe-se que o guaco (Mikania glomerata) tem propriedades broncodilatadoras (Lopes, 1997), antiinflamatórias (Falcão et al., 2005), antidiarréica (Salgado et al., 2005), antimicrobiana (Pessini et al., 2003; Do Amaral et al., 2003; Dos Santos et al., 2003) e atividade IMAO (Do Amaral et al., 2003). Contudo, poucos estudos atestam suas propriedades antialérgicas. Entretanto, uma fração chamada de MG1 obtida do extrato etanólico das folhas do guaco foi administrada em ratos no modelo de pleurisia alérgica após indução de ovoalbumina intrapleural. Como resultado, observouse a inibição da degranulação mastocitária induzida por 
antígenos, entre outros efeitos igualmente relevantes, oferecendo, assim, suporte à utilização popular do guaco no combate às alergias respiratórias (Fierro et al., 1999).

O óleo de copaíba é um líquido transparente utilizado popularmente como antiinflamatório (Cascon; Gilbert, 2000), em afecções pulmonares (tosses e bronquites, gripes e resfriados) (Paiva et al., 1998), antiasmático, expectorante, na pneumonia e sinusite (Veiga-Júnior; Pinto, 2002). Até o momento, não foram encontrados estudos clínicos utilizando óleo de copaíba, porém alguns trabalhos sobre a atividade biológica em animais foram recentemente publicados, como atividade antitumoral (Lima et al., 2003), efeito protetor de colite aguda e do dano intestinal (Paiva et al., 2004a,b) e antiinflamatório (Falcão et al., 2005). Alguns trabalhos analíticos recentes têm sido realizados buscando a padronização desta matéria prima, apesar de suas variações naturais (Biavatti et al., 2006).

A própolis e o mel são usados, em muitos países, para o tratamento de infecções das vias aéreas. Em modelos in vitro, extratos de própolis inibiram a agregação plaquetária e a síntese de eicosanóides, sugerindo propriedades antiinflamatórias (Khayyal et al., 1993; Paulino et al., 2003). Amostras de mel e própolis produzidos pela Apis mellifera já mostraram atividade contra S. aureus (Miorin et al., 2003; Do Amaral et al., 2003; Dos Santos et al., 2003). Destaca-se o efeito inibidor do mel de abelhas contra bactérias dos gêneros Staphylococcus, Streptococcus e Bacillus (Cummings et al., 1989; Shamala et al., 2000; Adeleye; Opiah, 2003).

O objetivo do presente estudo foi avaliar a toxicologia clínica do xarope Saratosse ${ }^{\circledR} \mathrm{em}$ vários órgãos e sistemas. Para tanto, procuramos identificar possíveis eventos adversos e avaliar alterações laboratoriais, de significado clínico, que denotassem comprometimento hematológico, metabólico, renal, hepático e cardiovascular em voluntários adultos sadios de ambos os sexos.

\section{MATERIAL E MÉTODOS}

\section{Desenho experimental}

O desenho consistiu de um estudo não controlado do tipo aberto, com 26 voluntários sadios, adultos, sendo 13 homens e 13 mulheres. Neste estudo, cada voluntário atuou como seu próprio controle, uma vez que seus próprios dados coletados no período de pré-estudo seriam comparados com os obtidos durante e após o tratamento.

\section{Seleção de voluntários}

No período que precedeu o estudo (pré-estudo), os voluntários, que deveriam ter idade entre 18 e 50 anos, foram submetidos a exames clínico e complementares para atestar seu estado de higidez, avaliando-se suas funções hepática, renal e cardiorrespiratória.

Do exame clínico constaram história médica e familiar, exame físico, aferição dos sinais vitais e cálculo do Índice de Massa Corpórea, compulsoriamente entre 19 e $30 \mathrm{~kg} / \mathrm{m}^{2}$. Também foram realizados eletrocardiograma (ECG) e uma extensa avaliação laboratorial que incluía análises hematológica, bioquímica e sorológica. A mesma avaliação, excetuando-se os testes sorológicos, foi repetida após cada semana de tratamento e 7 dias após a última administração.

Os candidatos que usaram qualquer fármaco 7 dias antes do início do estudo ou participaram de qualquer outro nos três meses que o precederam foram excluídos. $\mathrm{O}$ mesmo aconteceu àqueles que perderam $450 \mathrm{~mL}$ ou mais de sangue 3 meses antes ou que faziam abuso de álcool ou drogas.

\section{Composição do fitoterápico}

Saratosse ${ }^{\circledR}$ contém concentrações padronizadas de cada substância ativa das plantas utilizadas. Cada 100 $\mathrm{g}$ do produto contém extrato fluido de Mikania glomerata $(5,0 \mathrm{~g})$, tintura de própolis $(1,5 \mathrm{~g})$, essência de Mentha piperita $(0,3 \mathrm{~g})$, óleo essencial de Eucalyptus globulus $(0,3 \mathrm{~g})$, oleorresina de Copaifera multijuga $(0,1 \mathrm{~g})$, solução de sorbitol $(70,0 \mathrm{~g})$ e mel de abelhas q.s.p.

\section{Administração do fitoterápico}

Os voluntários compareceram diariamente, durante 28 dias ininterruptos, à Unidade de Farmacologia Clínica na Universidade Federal do Ceará, onde receberam uma dose oral de $15 \mathrm{~mL}$ de Saratosse ${ }^{\circledR}$ (lote E), fornecido pela Floramel Indústria e Comércio Ltda. (Brasil), quatro vezes ao dia, seguido de ingestão de $200 \mathrm{~mL}$ de água. Os horários de administração, sinais vitais (pressão arterial e pulso), bem como todas intercorrências ocorridas durante o estudo, foram devidamente registrados nos Formulários para Relato de Caso. Os voluntários permaneceram em suas atividades rotineiras, mantendo, também, suas dietas habituais.

\section{Eventos adversos}

Os voluntários foram orientados a relatar qualquer evento adverso e/ou utilização de alguma medicação adicional, para que houvesse acompanhamento adequado. Esses foram classificados quanto à intensidade como leve, moderado e severo. Quanto à relação de causalidade, foram utilizados os seguintes critérios: atribuído, provável, possível e não atribuído (Tavares, 2005).

\section{Aspectos éticos}

Os voluntários receberam explicação sobre a natureza e os objetivos do estudo. Após terem suas dúvidas sanadas, os voluntários, se de acordo com o protocolo clínico, assinaram o Termo de Consentimento 
Livre e Esclarecido - condição imprescindível para que fossem integrados.

O projeto de pesquisa, o protocolo experimental e o termo de consentimento livre e esclarecido foram aprovados pelo Comitê de Ética em Pesquisa da UFC, credenciado pela Comissão Nacional de Ética em Pesquisa do Conselho Nacional de Saúde/ Ministério da Saúde. O estudo obedeceu as normas estabelecidas na Resolução 196/96 (CNS), que regulamenta a pesquisa em seres humanos e na Resolução 251/97 (CNS), que normatiza os testes clínicos de novos medicamentos (Tavares, 2005).

\section{Análise estatística}

A análise estatística dos dados foi feita por meio da Análise de Variância (ANOVA) seguida do teste de Dunnett, obtendo-se, assim, a menor diferença significante ( $p<0,05)$, utilizando-se Microsoft Excel Version 7.0 e Graph Pad Prism Version 3.02. Os dados iniciais (préestudo) foram comparados com aqueles obtidos durante e após o término do ensaio (pós-estudo).

\section{RESULTADOS E DISCUSSÃO}

Os valores, para pulso radial e pressão diastólica de ambos os grupos, não variaram. A pressão sistólica dos homens, no entanto, decresceu no pós-estudo (Tabela 1), sendo este dado compatível com dados sobre a atividade diurética da Copaifera multijuga e da Mentha piperita (Fernández; Pereira, 1989; Della Loggia; Tubaro, 1990) e efeito vasodilatador do extrato de Mikania glomerata (Moura et al., 2002). Porém, sabe-se que variações na pressão arterial podem ser fisiológicas e ocorrer de acordo com o estado de movimento/repouso, estresse e condições nutricionais; logo, a alteração ocorrida pode não ser devido ao uso do fitoterápico. Além disso, os valores obtidos estiveram dentro da faixa de normalidade proposta para o estudo, assim como aqueles obtidos para o pulso radial. Por isso, esta alteração não constitui em indício de cardiotoxicidade, indo de encontro à ação deletéria atribuída ao felandreno encontrado no Eucalyptus globulus (Simões et al., 1999; Alonso, 1998).
A dosagem da hemoglobina e a contagem dos leucócitos não revelaram diferenças significativas sob o ponto de vista estatístico (Figura 1). Nada foi encontrado na literatura a respeito da possibilidade de alterações no hemograma de indivíduos sadios, após o uso das plantas medicinais utilizadas na elaboração da formulação, assim como mel e própolis.

Apesar da ação diurética atribuída à Copaifera multijuga (Fernández; Pereira, 1989) e à Mentha piperita (Della Loggia; Tubaro, 1990), a determinação dos índices de sódio não evidenciou alterações estatísticas no grupo dos homens em relação ao pré-estudo. Quanto ao grupo das mulheres, observou-se um pequeno decréscimo, compatível com a literatura, do sódio sérico nas segunda, terceira e quarta semana e no pós-estudo (Figura 2A).

A dosagem de potássio do grupo dos voluntários do sexo masculino indicou redução ao fim da primeira semana de tratamento em relação ao pré-estudo. Adosagem das voluntárias não variou. $\mathrm{O}$ resultado confirma o efeito diurético não associado a uma espoliação significativa de potássio da Mentha piperita, obtido por Della Loggia e Tubaro, em 1990 (Figura 2B).

$\mathrm{O}$ aumento da ingestão de água e sal na dieta pode influenciar tais dosagens. Sugere-se, então, que o uso do fitoterápico não causou o aparecimento de alterações clínicas nos níveis séricos dos eletrólitos, apesar das variações estatísticas, estando os índices dentro da faixa de normalidade.

O valor sérico da creatinina, além da função renal, depende da massa muscular, nutrição e ocorrência de edema (Soares et al., 2002). A determinação da concentração de creatinina sérica não mostrou variação estatística em nenhum período analisado para ambos os grupos. Portanto, os resultados confirmam a nãoocorrência de alterações metabólicas ou cárdio-renais, uma vez que os índices estiveram dentro dos limites de normalidade. Não foram encontrados achados que relacionassem o uso das substâncias contidas com os níveis séricos de creatinina (Figura 2C).

Apesar da ingestão de mel incorporado ao fitoterápico, a glicemia dos voluntários foi reduzida ao final das semanas de tratamento, excetuando-se a terceira (Figura 2D). Os resultados apontam para influência do

Tabela 1. Análise da pressão arterial e do pulso aferidos (média \pm DP).

\begin{tabular}{|c|c|c|c|c|c|c|c|}
\hline & & Pré - Estudo & 1a Semana & 2a Semana & 3a Semana & 4a Semana & Pós - Estudo \\
\hline $\begin{array}{l}\text { Pressão } \\
\text { Sistólica } \\
(\mathrm{mmHg})\end{array}$ & $\begin{array}{l}\mathrm{H}: \\
\mathrm{M}:\end{array}$ & $\begin{array}{l}123,08 \pm 7,23 \\
111,15 \pm 9,16\end{array}$ & $\begin{array}{r}117,69 \pm 7,25 * \\
116,15 \pm 9,39\end{array}$ & $\begin{array}{l}116,92 \pm 8,30 * \\
113,46 \pm 5,91\end{array}$ & $\begin{array}{l}116,15 \pm 6,50^{*} \\
113,08 \pm 10,32\end{array}$ & $\begin{array}{l}120,38 \pm 7,49 \\
113,46 \pm 10,49\end{array}$ & $\begin{array}{l}113,85 \pm 8,70^{*} \\
106,54 \pm 12,48\end{array}$ \\
\hline $\begin{array}{l}\text { Pressão } \\
\text { Diastólica } \\
(\mathrm{mmHg})\end{array}$ & $\begin{array}{l}\mathrm{H}: \\
\mathrm{M}:\end{array}$ & $\begin{array}{l}80,00 \pm 9,13 \\
70,77 \pm 8,38\end{array}$ & $\begin{array}{l}76,92 \pm 6,30 \\
76,92 \pm 8,30^{*}\end{array}$ & $\begin{array}{l}78,85 \pm 8,20 \\
74,62 \pm 5,58^{*}\end{array}$ & $\begin{array}{l}76,15 \pm 6,50 \\
72,31 \pm 5,99\end{array}$ & $\begin{array}{l}78,46 \pm 8,01 \\
78,08 \pm 14,80^{*}\end{array}$ & $\begin{array}{c}76,92 \pm 4,80 \\
75,77 \pm 9,54^{*}\end{array}$ \\
\hline $\begin{array}{l}\text { Pulso } \\
(\mathrm{mmHg})\end{array}$ & $\begin{array}{l}\mathrm{H}: \\
\mathrm{M}:\end{array}$ & $\begin{array}{l}73,69 \pm 5,59 \\
73,23 \pm 9,04 \\
\end{array}$ & $\begin{array}{r}68,62 \pm 5,38^{*} \\
75,08 \pm 6,96\end{array}$ & $\begin{array}{l}69,85 \pm 7,05^{*} \\
73,54 \pm 8,09 \\
\end{array}$ & $\begin{array}{l}71,08 \pm 8,82 \\
75,38 \pm 5,38\end{array}$ & \begin{tabular}{|l|}
$72,31 \pm 5,53$ \\
$74,77 \pm 8,55$ \\
\end{tabular} & $\begin{array}{l}73,85 \pm 9,47 \\
75,85 \pm 10,85\end{array}$ \\
\hline
\end{tabular}

H: Homem, M: Mulher; * Diferença estatisticamente significante do pré-estudo $(\mathrm{p}<0,05)$.

\begin{tabular}{r|r} 
& \\
\hline Rev. Bras. Farmacogn. & 352 \\
Braz J. Pharmacogn. & \\
16(3):jul/set. 2006 &
\end{tabular}



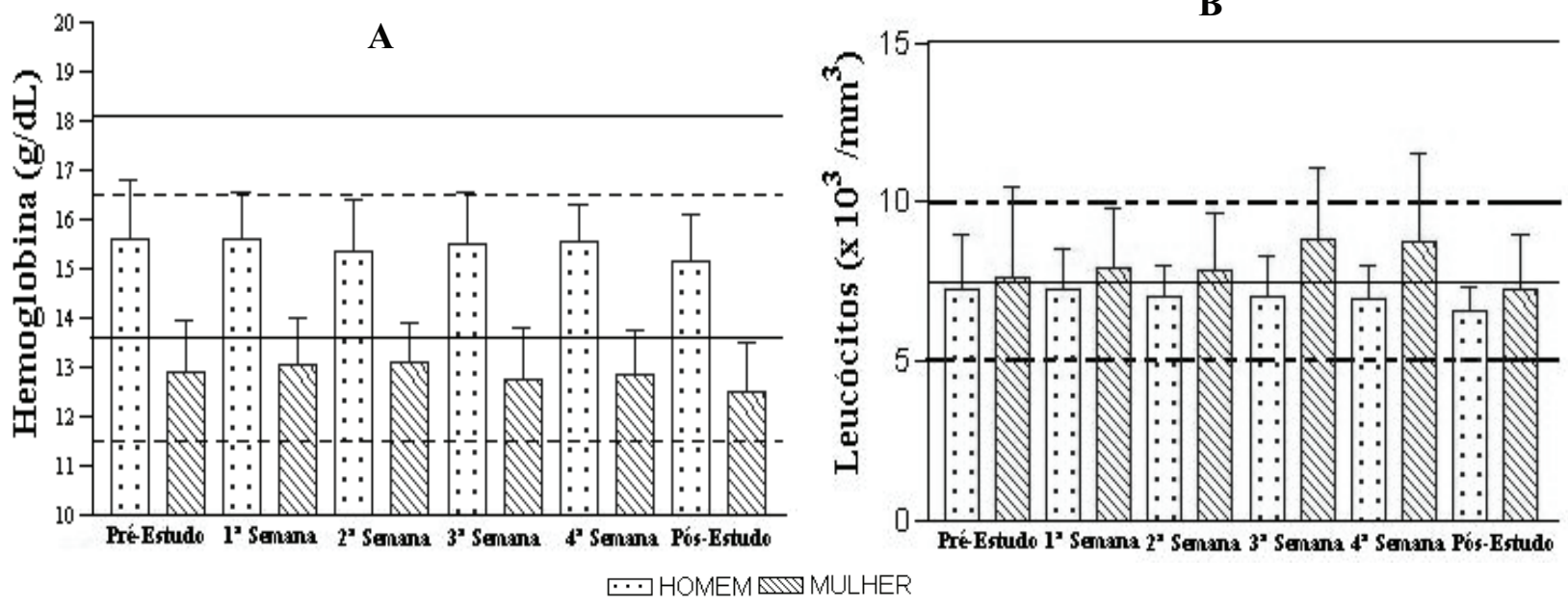

Figura 1. Análise da função hematológica, ${ }^{*} \mathrm{p}<0,05$. Valores de referência: A: Hemoglobina (— Homem: 13,5 a $18 \mathrm{~g} / \mathrm{dL}$; - Mulher: 11,5 a 16,5 g/dL), B: Leucócitos (—-- — Homem e Mulher: 5 a 10 g/dL).
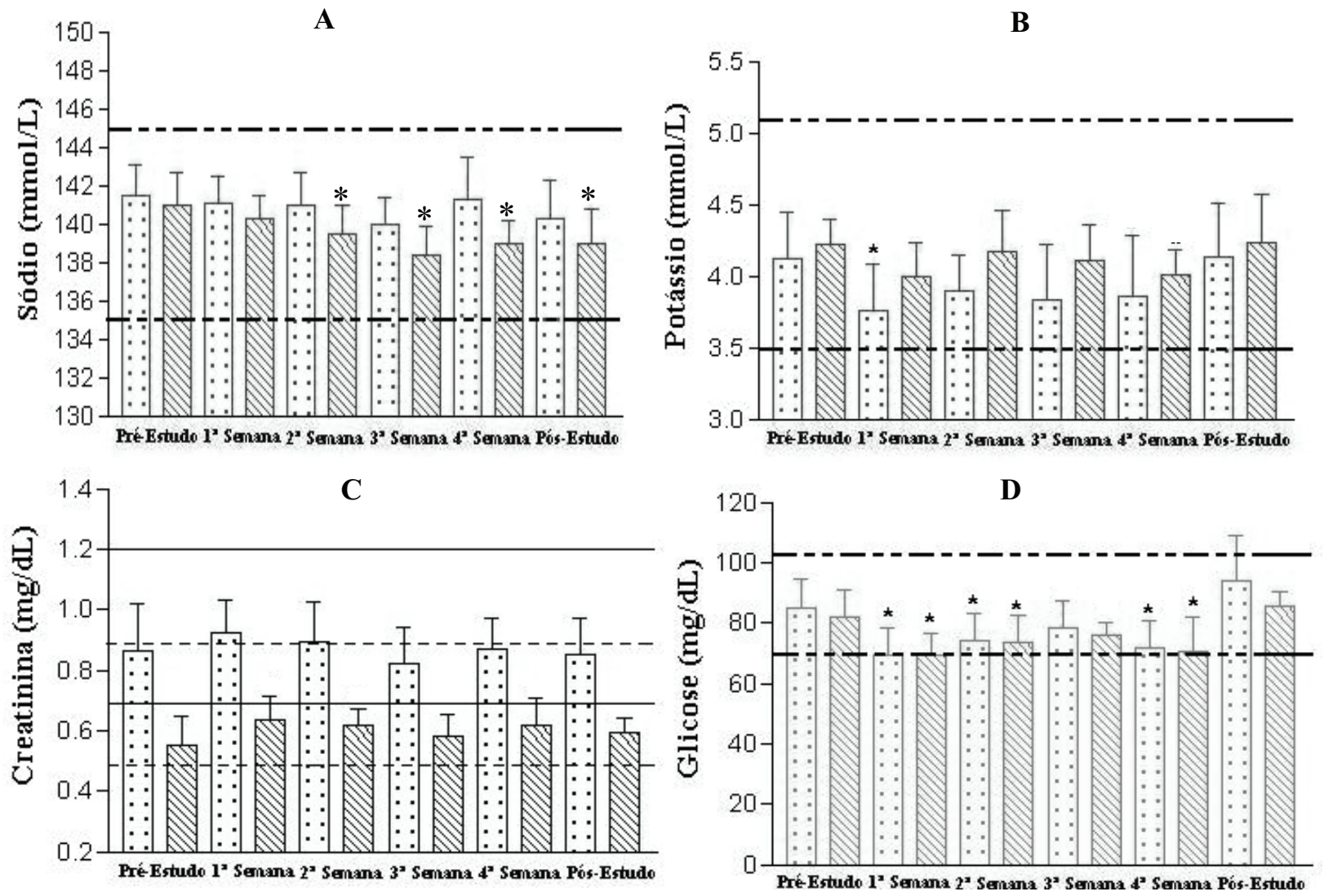

Figura 2. Análise dos eletrólitos e das funções renal e metabólica, ${ }^{*} \mathrm{p}<0,05$. Valores de referência: A: Sódio (- -- — Homem e Mulher: 135 a $145 \mathrm{mmol} / \mathrm{L})$; B: Potássio (— -- — Homem e Mulher: 3,5 a 5,1 mmol/L); C: Creatinina (— Homem: 0,7 a 1,2 mg/dL; - - Mulher: 0,5 a 0,9 mg/dL.); D: Glicose (— -- — Homem e Mulher: 70 a $105 \mathrm{mg} / \mathrm{dL}$ ). 


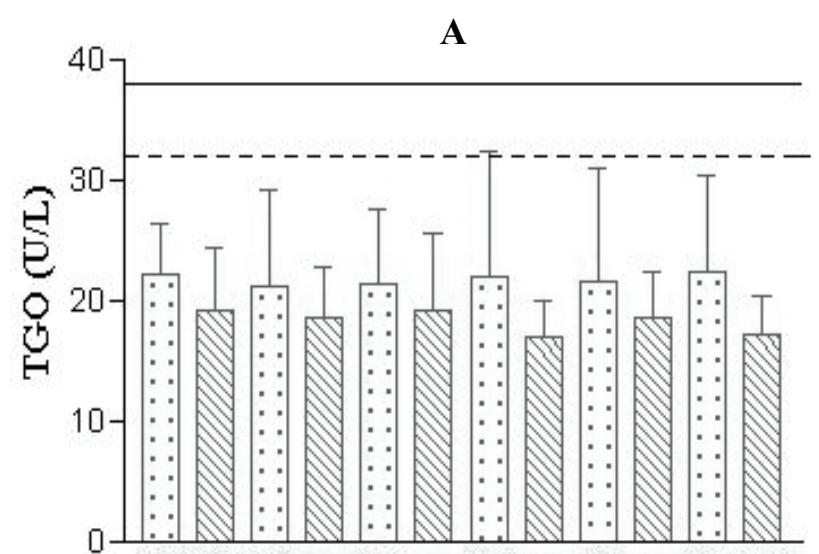

Prée Estudo $1^{x}$ Senana $2^{x}$ Senana $3^{x}$ Senana $4^{x}$ Senana Pós-Estudo
B

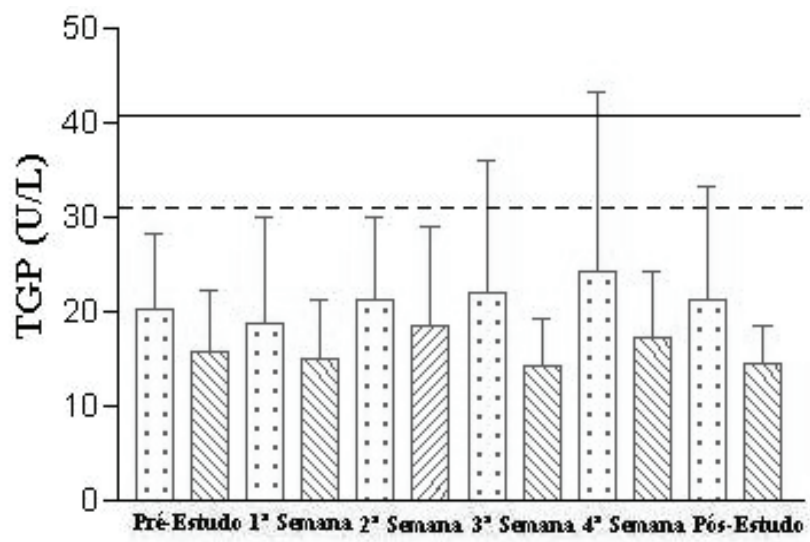

‥ HOMEM MUV MULHER

Figura 3. Análise da função hepática, *p <0,05. Valores de referência: A: TGO (— Homem: $\leq 38$ U/L; - - Mulher: $\leq 32$ U/L), B: TGP ( - Homem: $\leq 41 \mathrm{U} / \mathrm{L} ;$ - - Mulher: $\leq 31 \mathrm{U} / \mathrm{L})$.

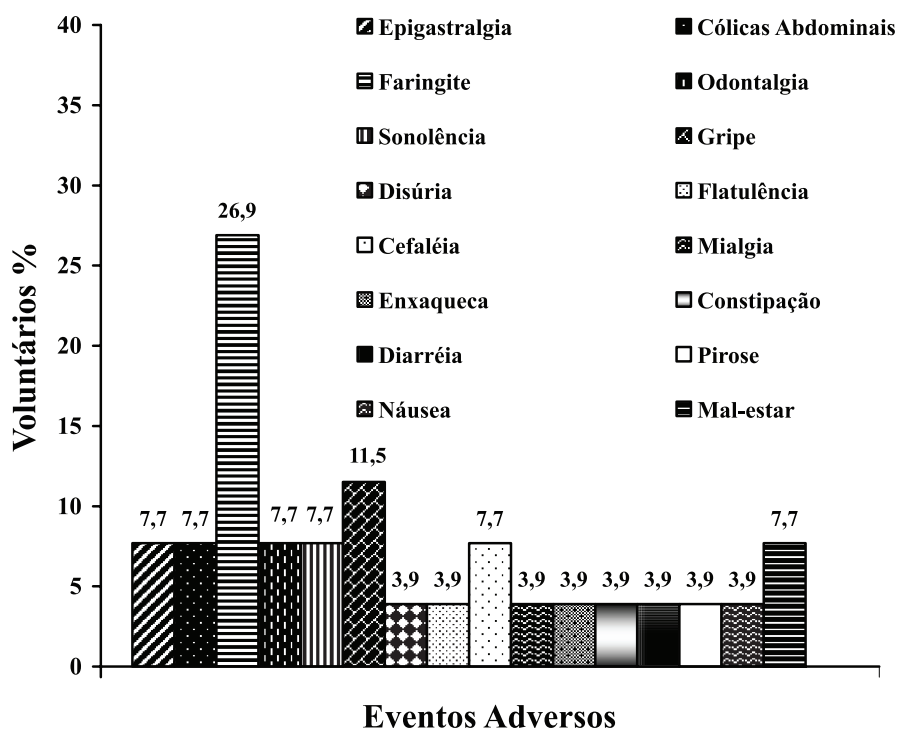

Figura 4. Eventos adversos relatados.

extrato de folhas de Eucalyptus globulus, que parece exercer ação hipoglicemiante em modelos animais (Handa; Chawla-Maninder, 1989; Barbosa-Filho et al., 2005). As oscilações experimentadas ocorreram dentro da faixa de valores esperados, não tendo significado clínico.

$\mathrm{O}$ uso da formulação parece não ter afetado os índices hepáticos (Figura 3), pois os voluntários não apresentaram queixas compatíveis com doenças hepáticas, de acordo com o exame clínico realizado. Além disso, a despeito dos possíveis distúrbios gastrintestinais causados pela Copaifera multijuga e pelo Eucalyptus globulus (Basile et al., 1988; Newall et al., 1996), não figuram entre os dados consultados da literatura, registros de discrepâncias nos níveis das enzimas hepáticas causadas pelas espécies envolvidas na formulação do fitoterápico avaliado.

Durante o estudo, foi observada a incidência de um caso de diarréia, pirose e náuseas (Figura 4). Os episódios de diarréia e pirose foram classificados como possivelmente atribuídos ao medicamento, uma vez que pode ocorrer diarréia em casos de ingestão de doses altas de oleorresina de Copaifera multijuga (Basile et al., 1988) e pirose é possível porque o mentol pode causar desconforto gástrico (Gattusso et al., 1991). O episódio de náusea também recebeu a mesma classificação, pois foi descrito em casos de administração de doses altas de óleo de Eucalyptus globulus (Newall et al., 1996).

Também foram reportados um caso de disúria, 
flatulência, mialgia, enxaqueca e constipação; dois casos de dor abdominal, odontalgia, cefaléia, sonolência e malestar; três casos de gripe e sete de faringite. O relato de sonolência é compatível com o dado prévio de que a Copaifera multijuga possa exercer uma ação depressora central (Basile et al., 1988), fazendo deste um sintoma possivelmente atribuído ao medicamento. Não foram encontrados dados, na literatura, que atestem que qualquer um dos componentes possa provocar os demais eventos descritos. Além disso, não há evidências que comprovem a relação de causalidade entre estes e o fitoterápico, e há, ainda, o indício de fatores alternativos podem ter desencadeado os mesmos. Por estas razões, os episódios supra-enumerados não foram atribuídos ao seu uso.

Os sintomas gripe e faringite chamaram a atenção dos pesquisadores, dada a indicação do fitoterápico para as afecções do trato respiratório. Os voluntários que apresentaram tais sintomas não se queixaram de tosse ou produção purulenta. Também foi verificado que nenhum deles cursou com agravamento do seu estado, apresentando melhoria de suas condições em dias próximos.

Houve dois casos de epigastralgia, que pode ser causada por altas doses de óleo de Eucalyptus globulus (Newall et al., 1996). Apesar da baixa concentração na formulação, por estar previsto na literatura, o episódio foi classificado como possivelmente atribuído.

Os eventos adversos relatados foram classificados quanto à intensidade como leves, por serem facilmente tolerados e por não interferirem nas suas atividades cotidianas.

Os resultados alcançados possibilitam avaliar, no futuro, a eficácia terapêutica deste fitoterápico, e também, desenvolver indispensáveis estudos de farmacocinética (Tavares, 2005). É de suma relevância conhecer de que forma e quanto das substâncias ativas: a) atuarão em um sítio para desencadear as ações farmacológicas desejadas; b) serão absorvidas; c) serão metabolizadas; d) serão excretadas. Também interessa conhecer que possíveis interações poderão ocorrer com o uso do fitoterápico em questão e isso só é possível após a avaliação da sua segurança.

Por meio dos resultados apresentados, infere-se que a avaliação das funções analisadas dos voluntários após tratamento com o fitoterápico Saratosse ${ }^{\circledR}$, via oral, na dose de $15 \mathrm{~mL}$, quatro vezes ao dia, durante 28 dias, não apontou sinais de toxicidade sistêmica. Os dados colhidos através dos exames encontraram-se dentro dos limites de normalidade ou apresentaram alterações discretas sem significância clínica, não indicando comprometimento sistêmico. Os eventos adversos relatados por voluntários pareceram não ter relação de causalidade com o fitoterápico.

\section{AGRADECIMENTOS}

Este estudo contou com o apoio da Coordenação de Aperfeiçoamento de Pessoal de Nível Superior (CAPES), Conselho Nacional de Desenvolvimento Científico e Tecnológico (CNPq), Fundação Cearense de Amparo à Pesquisa (FUNCAP) e do Instituto Claude Bernard (InCB).

\section{REFERÊNCIAS}

Adeleye IA, Opiah L 2003. Antimicrobial activity of extracts of local cough mixtures on upper respiratory tract bacterial pathogens. West Indian Med J 52: 188190.

Alonso JR 1998. Tratado de Fitomedicina. Buenos Aires: Isis Ediciones SRL.

Barbosa-Filho JM, Vasconcelos THC, Alencar AA, Batista LM, Oliveira RAG, Guedes DN, Falcão HS, Moura MD, Diniz MFFM, Modesto-Filho J. 2005. Plants and their active constituents from South, Central, and North America with hypoglycemic activity. Rev Bras Farmacogn 15: 392-413.

Basile AC, Sertié JAA, Freitas PCD, Zanini AC 1988. Antiinflammatory activity of oleoresin from Brazilian Copaifera. J Ethnopharmacol 22: 101-109.

Biavatti MW, Dossin D, Deschamps FC, Lima MP 2006. Análise de óleos-resinas de copaíba: contribuição para o seu controle de qualidade. Rev Bras Farmacogn 16: 230-235.

Cascon V, Gilbert B 2000. Characterization of the chemical composition of oleoresins of Copaifera guianensis Desf., Copaifera duckei Dwyer and Copaifera multijuga Hayne. Phytochemistry 55: 773-778.

Cummings JH, Macfarhana GT, Draser BS 1989. The gut microflora and its significance. In: Whitehead, R. (org.), Gastrointestinal and Oesophagal Pathology. London: Churchill Livingston, p.201-219.

Della Loggia R, Tubaro A 1990. Evaluation of some pharmacological activities of a peppermint extract. Fitoterapia 61: 215-221.

Do Amaral RR, Arcenio Neto F, Carvalho ES, Teixeira LA, De Araújo GL Sharapin N Testa B, Gnerre C, Rocha L 2003. Avaliação da atividade IMAO e antibacteriana de extratos de Mikania glomerata Sprengel. Rev Bras Farmacogn 13: 24-27.

Dos Santos CR, Arcenio F, Carvalho ES, Lúcio EMRA, Araújo GL, Teixeira LA, Sharapin N, Rocha L 2003. Otimização do processo de extração de própolis através da verificação da atividade antimicrobiana. Rev Bras Farmacogn 13 (Supl. 1): 71-74.

Falcão HS, Lima IO, Santos VL, Dantas HF, Diniz MFFM, Barbosa-Filho JM, Batista LM 2005. Review of the plants with anti-inflammatory activity studied in Brazil. Rev Bras Farmacogn 15: 381-391.

Fernández R, Pereira N 1989. Copalic acid analgesic activity in mice. Simpósio Sino-Brasilenho de Química e Farmacologia de Produtos naturais. Rio de Janeiro, Brasil.

Fierro IM, Silva, ACB, Lopes CS, Moura RS, Barja-Fidalgo C 1999. Studies on the allergic activity of Mikania glomerata. J Ethnopharmacol 66: 19-24.

Gatusso P, Reddy V, Castelli M 1991. Exogenous lipoid pneumonitis due to Vicks Vaporoub (N.R.) inhalation diagnosed by fine needle aspiration 
cytology (letter). Cytopathology 2: 315-316.

Handa S, Chawla Maninder A 1989. Hypoglycaemic plants. A review. Fitoterapia 60: 205

Khayyal MT, El-Ghazaly MA, El-Khatib AS 1993. Mechanisms involved in the anti-inflammatory effect of propolis extract. Drug Exp Clin Res 19: 197-203.

Lima SR, Veiga-Júnior VF, Christo HB, Pinto AC, Fernandes PD 2003. In vivo and in vitro studies on the anticancer activity of Copaifera multijuga Hayne and its fractions. Phytother Res 17: 1048-1053.

Lopes CS 1997. Efeitos do guaco (Mikania glomerata) na musculatura lisa respiratória. XII Annual Meeting of FeSBE. Caxambú, Brasil.

Miorin PL, Levy Junior NC, Custodio AR, Brez WA, Marcucci MC 2003. Antibacterial activity of honey and propolis from Apis mellifera and T. angustula against Staphylococcus aureus. J Appl Microbiol 95: 913-920.

Moura RS, Costa SS, Jansen JM, Silva AC, Lopes CS, BernardoFilho M, Silva VN, Criddle DN, Portela BN, Rubenich LMS, Araújo RG, Carvalho LCRM 2002. Bronchodilatador activity of Mikania glomerata Sprengel on human bronchi and guinea-pig trachea. J Pharma Pharmacol 54: 249-256.

Newall C, Anderson L, Phillipson J 1996. Herbal Medicines a Guide for Health-Care Professionals. London: Pharmaceutical Press.

Paiva LA, Rao VS, Gramosa NV, Silveira ER 1998. Gastroprotective effect of Copaifera langsdorffi $i$ oleoresin on experimental gastric ulcer models in rats. J Ethnopharmacol 62: 73-78.

Paiva LA, Gurgel LA, De Sousa ET, Silveira ER, Silva RM, Santos FA, Rao VS 2004a. Protective effect of Copaifera langsdorffii oleo-resin against acetic acidinduced colitis in rats. $J$ Ethnopharmacol 93: 51-56.

Paiva LA, Gurgel LA, Campos AR, Silveira ER, Rao VS 2004b. Attenuation of ischemia/reperfusion-induced intestinal injury by oleo-resin from Copaifera langsdorffii in rats. Life Sci 75: 1979-1987.

Paulino N, Dantas AP, Bankova V, Longhi DT, Scremin A, Castro SL, Calixto JB 2003. Bulgarian propolis induces analgesic and anti-inflammatory effects in mice and inhibits in vitro contraction of airway smooth muscle. J Pharmacol Sci 93: 307-313.

Pereira RC, Oliveira MTR, Lemos GCS 2004. Plantas utilizadas como medicinais no município de Campos de Goytacazes-RJ. Rev Bras Farmacogn 14(Supl. 1): 37-40.

Pessini GL, Holetz FB, Sanches NR, Cortez DAG, Dias-Filho BP, Nakamura CV 2003. Avaliação da atividade antibacteriana e antifúngica de extratos de plantas utilizados na medicina popular. Rev Bras Farmacogn 13(Supl. 1): 21-24.

Reis CMF, Carvalho JCT, Caputo LRG, Patrício KCM 2000. Atividade antiinflamatória, antiúlcera gástrica e toxicidade subcrônica do extrato etanólico de própolis. Rev Bras Farmacogn 9/10: 43-52.

Salgado HRN, Roncari AFF, Moreira RRD 2005. Antidiarrhoeal effects of Mikania glomerata Spreng. (Asteraceae) leaf extract in mice. Rev Bras Farmacogn 15: 205208.

Shamala TR, Shri Jyothi Y, Saibaba P 2000. Stimulatory effect of honey on multiplication of lactic acid bacteria under in vitro and in vivo conditions. Lett Appl Microbiol 30: 453-455.

Simões CMO, Schenkel EP, Gosmann G, Mello JCP, Mentz LA, Petrovick PR 1999. Farmacognosia: da planta ao medicamento. Florianópolis/Porto Alegre: Editora da UFSC/Editora da UFRGS

Soares JLMF, Pasqualotto AC, Rosa DD, Leite VRS 2002. Métodos Diagnósticos - Consulta Rápida. Porto Alegre: Artmed Editora.

Tavares JP 2005. Estudo de toxicologia clínica de três fitoterápicos à base de associações de plantas, mel e própolis em voluntários sadios. Fortaleza, p. 3940 e 78. Dissertação de Mestrado - Programa de Pós-Graduação em Farmacologia, Universidade Federal do Ceará.

Veiga-Júnior VF, Pinto AC 2002. O gênero Copaifera L. Quim Nova 25: 273-286.

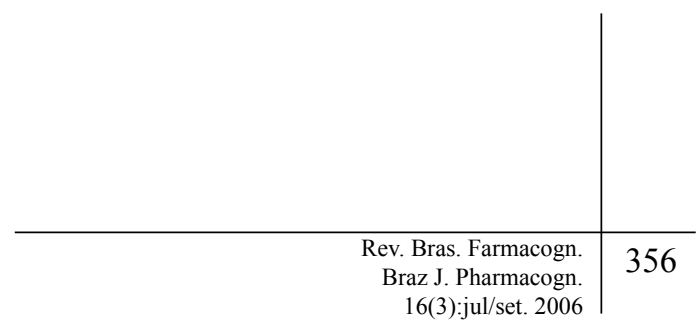

